\title{
Impact of the Polymorphism rs9264942 near the HLA-C Gene on HIV-1 DNA Reservoirs in Asymptomatic Chronically Infected Patients Initiating Antiviral Therapy
}

\author{
Laura Herráiz-Nicuesa, ${ }^{1,2}$ Diana Carolina Hernández-Flórez, ${ }^{1,2}$ Lara Valor, ${ }^{1,2}$ \\ Sonia García-Consuegra, ${ }^{1,2}$ Juan Paulo Navarro-Valdivieso, ${ }^{1,2}$ Eduardo Fernández-Cruz, ${ }^{1,2}$ \\ and Carmen Rodríguez-Sainz ${ }^{1,2}$
}

${ }^{1}$ Servicio de Inmunología Clínica, Hospital General Universitario Gregorio Marañón, Madrid, Spain

${ }^{2}$ Instituto de Investigación Sanitaria Gregorio Marañón, Madrid, Spain

Correspondence should be addressed to Carmen Rodríguez-Sainz; crodriguezs@salud.madrid.org

Received 28 July 2017; Accepted 17 October 2017; Published 28 December 2017

Academic Editor: Daniel Lamarre

Copyright (c) 2017 Laura Herráiz-Nicuesa et al. This is an open access article distributed under the Creative Commons Attribution License, which permits unrestricted use, distribution, and reproduction in any medium, provided the original work is properly cited.

\begin{abstract}
Several genome-wide association studies have identified a polymorphism located $35 \mathrm{~kb}$ upstream of the coding region of $H L A$ - $C$ gene ( $r$ 9264942; termed $-35 \mathrm{C} / \mathrm{T}$ ) as a host factor significantly associated with the control of HIV-1 viremia in untreated patients. The potential association of this host genetic polymorphism with the viral reservoirs has never been investigated, nor the association with the viral control in response to the treatment. In this study, we assess the influence of the polymorphism $-35 \mathrm{C} / \mathrm{T}$ on the outcome of virus burden in 183 antiretroviral-naive HIV-1-infected individuals who initiated antiviral treatment (study STIR-2102), analyzing HIV-1 RNA viremia and HIV-1 DNA reservoirs. The rs9264942 genotyping was investigated retrospectively, and plasma levels of HIV-1 RNA and peripheral blood mononuclear cell- (PBMC-) associated HIV1 DNA were compared between carriers and noncarriers of the protective allele $-35 \mathrm{C}$ before antiretroviral therapy (ART), one month after ART and at the end of the study (36 months). HIV-1 RNA and HIV-1 DNA levels were both variables significantly different between carriers and noncarriers of the allele $-35 \mathrm{C}$ before ART. HIV-1 DNA levels remained also significantly different one month posttherapy. However, this protective effect of the $-35 \mathrm{C}$ allele was not maintained after long-term ART.
\end{abstract}

\section{Introduction}

The clinical outcome of HIV-1 infection is highly variable and determined by complex interactions between virus, host, and environment. Part of this epidemiological heterogeneity could be attributed to host genetic factors, which have been extensively studied using whole genome approaches (reviewed in [1, 2]). Several genome-wide association studies (GWAS) have identified a polymorphism located $35 \mathrm{~kb}$ upstream of the coding region of the gene HLA-C (-35 C/T; rs9264942) as a host factor significantly associated with the control of HIV-1 viremia. Fellay et al. reported the first AIDS GWAS in 2007 [3]. Their study tracked the viral set point (mean plasma RNA level over several months once the immune system has settled to a steady-state level after primo-infection) in 486 European AIDS patients (Cohort EURO-CHAVI) that were genotyped. The variant $r s 9264942$ was the second most significant independent hit in the EURO-CHAVI study and resulted the top hit in the International HIV Controller Study involving 974 controllers, as reported three years later [4]. Another HIV-1 viral set point GWAS with 2554 Caucasian participants provided overwhelming confirmation of the SNP rs9264942 [5]. This SNP also associates strongly with differences in $H L A-C$ expression levels. The protective allele $(-35 C)$ leads to a lower viral load and is associated with higher expression of the HLA-C gene [3].

Recently, more work has focused on elucidating the functional significance of the $-35 \mathrm{C} / \mathrm{T} \mathrm{SNP}$, and several groups now have demonstrated HLA-C surface expression to be a 
TABLE 1: Baseline characteristics for the cohort of patients (study STIR-2102).

\begin{tabular}{lccc}
\hline & ART + HIV-immunogen & ART + placebo & Overall \\
\hline Number of subjects & 86 & 97 & 183 \\
Age, mean (S.D.) (years) & $35(8)$ & $33(7)$ & $34(7)$ \\
Gender, $N$ (\%) & & & \\
$\quad$ Male & $61(70.9)$ & $65(67.0)$ & 126 \\
$\quad$ Female & $25(30.0)$ & $32(33.0)$ & 57 \\
CD4 ${ }^{+}$T cells, mean (S.D.), cells $\times 10^{-6} \mathrm{~L}^{-1}$ & $405.1(72.1)$ & $401.0(74.9)$ & $402.9(73.4)$ \\
HIV-1 RNA, median (range), copies per $\mathrm{mL}$ & $14,150(459,801)$ & $14,650(243,801)$ & $14,525(459,801)$ \\
\hline
\end{tabular}

S.D.: standard deviation.

key element in the control of HIV viral load [6]. HLA-C surface expression has been correlated with the presence of microRNA binding sites that affect $H L A-C$ expression and control of HIV disease. It has been identified a binding site for the microinterference RNA, miR-148a, that is present in the sequence of some $H L A-C$ alleles, but missing in others due to polymorphism in the region. This polymorphism includes an insertion/deletion variant at the position 263 of the $3^{\prime}$ UTR of the HLA-C gene, which is in very strong linkage disequilibrium with rs9264942 in Caucasians [7]. The linkage disequilibrium of the -35 SNP with the $3^{\prime}$ UTR miRNA-148a binding site polymorphisms offers a functional explanation for the observed differences in HLA-C surface expression among individuals and the associated control of HIV disease. Globally, all these data support a role for $H L A-C$ in early and/or chronic infection $[8,9]$.

$H L A-C$ is the most recently evolved of the classical $M H C$ $I$ alleles and is restricted to humans and great apes $[10,11]$; and fewer alleles of $H L A-C$ have been identified. HLA-C plays a dual role in that it can present antigens to CTLs and it can inhibit natural killer (NK) cell (and possibly also CTL) lysis via its interaction with inhibitory receptors (killer immunoglobulin-like receptors, KIR). For reasons that are poorly understood, HLA-C is normally expressed on the cell surface at levels approximately 10 -fold less than most $H L A-A$ and $H L A-B$ allotypes [12-14]. In HIV-infected individuals, HLA-A and HLA-B molecules expressed on the surface of the infected cells are preferentially downmodulated by the viral accessory protein Nef, but not HLA-C molecules [15], and so HLA-C may have a unique role in presenting antigens to CTLs in HIV disease and modulating cytotoxicity.

Since the first GWAS study of Fellay, involving the variant $r s 9264942$ near the HLA-C as a genetic determinant in HIV-1 infection, most of the GWAS performed to date in cohorts involving various outcomes of HIV infection are focus on the viremia or the control of circulating virus and all of them have been designed in the absence of antiviral therapy. Viral burden has been extensively investigated in the HLA-C background analyzing plasmatic HIV-1 RNA, but HIV-1 reservoirs, particularly HIV-1 DNA levels are lesser known. The potential association of the variant rs9264942 with the viral control under antiviral treatment is also unknown.

The objective of this study was to assess the influence of the polymorphism rs 9264942 on the outcome of virus burden in 183 antiretroviral-naïve individuals who initiated antiviral treatment (study STIR-2102), analyzing HIV-1 RNA viremia and HIV-1 DNA reservoirs associated to PBMC. We quantified HIV-1 RNA from plasma and HIV-1 DNA obtained from PBMCs before antiretroviral therapy (ART) and during the treatment with a follow-up of 36 months.

\section{Materials and Methods}

2.1. Patients. One hundred eighty-three patients with asymptomatic HIV-1 chronic infection who had participated in the clinical trial STIR-2102 $[16,17]$ were retrospectively genotyped for the polymorphism rs9264942. The study STIR-2102 was a multicenter, randomized, double-blinded, placebo-controlled phase II clinical trial of antiretroviral therapy (ART) in combination with an HIV-1 immunogen (Remune $^{\circledR}$ ) in antiretroviral-naïve HIV-1-infected subjects with $\mathrm{CD}^{+}{ }^{+} \mathrm{T}$ lymphocytes between 300 and 700 cells $/ \mu \mathrm{L}$. ART consisted of zidovudine and didanosine. Patients started therapy one month prior randomization to receive ART plus the immunogen $(N=86)$ or ART plus placebo $(N=97)$. Treatment arms showed no differences in baseline factors, such as age, gender, risk group, viral load, and $\mathrm{CD}^{+} \mathrm{T}$ cells. The baseline characteristics for the study STIR-2102 are shown in Table 1. The immunovorological response to the therapy was similar at the end of the study with no significant differences between both arms. Prior to the commencement of the study STIR-2102, conducted between 1997 and 2001, Institutional Review Board approval by each participant hospital was obtained. Furthermore, informed consents from all participants, jointly with the review and approval of the protocol by the Spanish Agency of Medicament and Sanitary Products, were also obtained prior to the initiation of the trial.

2.2. Plasma HIV-1 RNA Viremia and $C D 4^{+}$T Cell Subset. Laboratory test including $\mathrm{CD}^{+}{ }^{+} \mathrm{T}$ cell counts and plasma HIV-1 RNA levels were carried out every three months throughout the study (36 months). Plasma levels of HIV-1 RNA were assessed using the Amplicor assay (Hoffman La Roche, Nutley, NJ) with a lower limit of quantification of 200 copies/mL (2.30 $\log _{10}$ copies/mL) [16, 17].

2.3. Polymorphism rs9264942 Genotyping Assessment. Blood samples were collected from patients during the trial, and DNA was isolated from the peripheral blood mononuclear cells by standard protocols. PBMC were separated by Ficoll gradient (Pharmacia, Uppsala, Sweden) and stored as 
TABLE 2: Frequencies of the genotypes for the SNP rs9264942 in the HLA-C 5 ' region, where $\mathrm{T}$ is the major allele and $\mathrm{C}$ is the minor allele. HIV-1 RNA and HIV-1 DNA levels are shown in the patients naïve for antiretroviral therapy (pre-ART), one month (1 m) post-ART, and at the end of the study (follow-up: 36 months).

\begin{tabular}{lcccccccc}
\hline \multicolumn{2}{c}{$\begin{array}{r}\text { rs9264942 } \\
\text { genotypes }\end{array}$} & Frequency & \multicolumn{2}{c}{ HIV-1 RNA, median (min-max) } & \multicolumn{3}{c}{ HIV-1 DNA, median (min-max) } \\
& $N(\%)$ & Pre-ART & 1 m post-ART & End of study & Pre-ART & 1 m post-ART & End of study \\
\hline \multirow{2}{*}{ TT } & \multirow{2}{*}{$54(29.5 \%)$} & 24,976 & 200 & 5400 & 927.5 & 1080 & 250 \\
Total & & & $(200-460,000)$ & $(200-60,000)$ & $(200-56,543)$ & $(250-32,560)$ & $(250-10,645)$ & $(250-2570)$ \\
$N=183$ & CT & $95(51.9 \%)$ & 11,450 & 200 & 1125 & 520.0 & 440 & 250 \\
& & & $(200-274,500)$ & $(200-25,000)$ & $(200-46,200)$ & $(250-12,985)$ & $(250-18,855)$ & $(250-10,120)$ \\
& CC & \multirow{2}{*}{$34(18.6 \%)$} & $(200-206,500)$ & $(200-16,100)$ & $(200-41,200)$ & $(250-17,425)$ & $(250-7475)$ & $(250-930)$ \\
& & & N.S. $p=0.068$ & N.S. & N.S. & $\boldsymbol{p}=\mathbf{0 . 0 0 1}$ & $\boldsymbol{p}=\mathbf{0 . 0 2 0}$ & N.S. \\
\hline
\end{tabular}

Median and minimum-maximum values of viremia and cellular-associated viral loads are indicated for the respective genotypes, HIV-1 RNA in copies/mL, and HIV-1 DNA in copies $/ 10^{6}$ PBMCs. The $p$ values are for median comparisons; N.S.: not significant.

dry pellets at $-80^{\circ} \mathrm{C}$. DNA was purified from each PBMC pellet by Wizard Genomic DNA Purification Kit (Promega Corporation, Madison, WI, USA), and DNA content was determined by spectrophotometric analysis.

The rs9264942 genotyping was retrospectively performed by real-time PCR amplification with the LightSNiP rs9264942 HLA-C (TIB MOLBIOL GmbH, Berlin, Germany; under license from Roche Diagnostics $\mathrm{GmbH}$ ) using LightCycler $^{\circledR}$ FastStart DNA Master HybProbe (Roche Diagnostics) and a LightCycler 1.5 Instrument, according to the manufacturer's protocol. The genotype was verified by sequencing.

2.4. HIV-1 DNA Level. HIV-1 DNA was quantified with a real-time quantitative PCR method using SYBR Green and primers of the pol gene as previously described [18]. Fluorescence was monitored specifically in a single point of each amplification cycle, allowing the determination of initial DNA copies through comparison with a standard curve constructed with DNA from the T-lymphoblastoid cell lines Jurkat and 8E5LAV that do not contain HIV-1 LAV or only in the amount of one single copy of HIV-1 LAV per cell, respectively. The detection limit of our assay was 250 copies $/ 10^{6}$ PBMC. Results of the PBMC-associated HIV-1 DNA in this cohort of patients were reported partially in a previous study [18].

2.5. Endpoints and Statistical Analysis. In STIR-2102 trial, the virologic endpoint was defined as time to the first increase of viral load above 5000 copies per milliliter and the immunological endpoint as time to the first decrease of $\mathrm{CD} 4^{+} \mathrm{T}$ cell count below 250 cells $/ \mu \mathrm{L}$ [16]. Subjects who at the end of the study at month 36 had not developed a primary endpoint were censored at the last available visit. Kaplan-Meier analysis was used to construct event-free survival curves, which were compared using the log-rank test. Adjusted hazard ratios (HRs) and the $95 \%$ confidence intervals (CIs) were calculated using multivariate Cox proportional-hazard models. Proportional hazard assumptions were assessed as previously described [19]. Virologic data did not show a normal distribution and were expressed as median. Mann-Whitney test was used to compare groups. We used the Statistical Package for the Social Sciences (SPSS) and Stata for statistical analysis.

\section{Results}

3.1. Distribution of the rs9264942 Genotypes in the Study Cohort. Among the 183 chronically infected study subjects, thirty-four (18.6\%) displayed the genotype homozygous for the protective allele $(-35 \mathrm{CC})$, whereas ninety-five $(51.9 \%)$ were heterozygous $(-35 C T)$ and fifty-four were homozygous for the allele $-35 \mathrm{~T}(29.5 \%)$ (Table 2). The allelic frequency of the minor allele $(-35 \mathrm{C})$ was $44.5 \%$. The study population was in Hardy-Weinberg equilibrium.

3.2. Association of the Allele -35 C with HIV-1 Viral Load. To evaluate the impact of the protective allele $-35 C$ on the steady-state plasma HIV-1 RNA levels in the study cohort, we compared viral load levels between carriers and noncarriers of the allele, at the baseline when individuals were ART-naïve. Patients included in this study were asymptomatic and maintained a relatively stable viral load off therapy. The analysis revealed decreased set points of the plasmatic viremia in $-35 C$ carriers as compared to those with the genotype $-35 T T$ reaching statistical significance $(p=0.035$; median comparison for independent samples). HIV-1 DNA levels were also significantly different between patients with and without the allele $-35 C(p<0.001$; median comparison for independent samples, Table 3). This allele was associated with lesser levels of HIV-1 DNA in PBMCs.

HIV-1 DNA levels in PBMCs remained also significantly different one month posttherapy [median $=425 \mathrm{cop} / 10^{-6}$ PBMCs $\left(\max =18,855, \min =250 \mathrm{cop} / 10^{-6} \mathrm{PBMCs}\right)$ in $-35 C$ carriers versus median $=1080 \mathrm{cop} / 10^{-6}$ PBMCs $\left(\max =10,645, \min =250 \mathrm{cop} / 10^{-6}\right.$ PBMCs $)$ in the individuals with the genotype -35 TT; $p=0.010$, median comparison for independent samples].

3.3. Association of the Allele $-35 \mathrm{C}$ with HIV-1 Disease Progression in Response to the Therapy. We did not find any protective effect of the allele $-35 \mathrm{C}$ in Kaplan-Meier survival analysis of the 183 therapy-naïve chronically infected individuals during the thirty-six months of the study STIR2102 after the initiation of the antiretroviral therapy. Carriers 
TABLE 3: Frequencies of the individuals carrying the protective allele rs9264942-35 C (genotypes CC + CT) versus noncarriers of this allele (genotype TT). HIV-1 RNA and HIV-1 DNA levels are shown in the patients naïve for antiretroviral therapy (pre-ART), one month $(1 \mathrm{~m})$ post-ART, and at the end of the study (follow-up: 36 months).

\begin{tabular}{|c|c|c|c|c|c|c|c|c|}
\hline \multirow{2}{*}{\multicolumn{2}{|c|}{$\begin{array}{c}\text { rs9264942 }(\mathrm{T}>\mathrm{C}) \\
-35 \mathrm{C} \text { allele (genotypes) }\end{array}$}} & \multirow{2}{*}{ Frequency $(\%)$} & \multicolumn{3}{|c|}{ HIV-1 RNA median (min-max) } & \multicolumn{3}{|c|}{ HIV-1 DNA median (min-max) } \\
\hline & & & Pre-ART & $1 \mathrm{~m}$ post-ART & End of study & Pre-ART & $1 \mathrm{~m}$ post-ART & End of study \\
\hline \multirow{3}{*}{$\begin{array}{l}\text { Total } \\
N=183\end{array}$} & Noncarriers (TT) & $54(29.5 \%)$ & $\begin{array}{c}24,976 \\
(200-460,000)\end{array}$ & $\begin{array}{c}200 \\
(200-60,000)\end{array}$ & $\begin{array}{c}3600 \\
(200-56,543)\end{array}$ & $\begin{array}{c}927,5 \\
(250-32,560)\end{array}$ & $\begin{array}{c}1080 \\
(250-10,645)\end{array}$ & $\begin{array}{c}250 \\
(250-2570)\end{array}$ \\
\hline & \multirow[t]{2}{*}{ Carriers $(\mathrm{CT}+\mathrm{CC})$} & \multirow[t]{2}{*}{$129(70.5 \%)$} & $\begin{array}{c}10,100 \\
(200-274,500)\end{array}$ & $\begin{array}{c}200 \\
(200-25,000)\end{array}$ & $\begin{array}{c}1730 \\
(200-132,000)\end{array}$ & $\begin{array}{c}500 \\
(250-17,425)\end{array}$ & $\begin{array}{c}425 \\
(250-18,855)\end{array}$ & $\begin{array}{c}250 \\
(250-15,920)\end{array}$ \\
\hline & & & $p=0.035$ & N.S. & N.S. & $p<0.001$ & $p=0.010$ & N.S. \\
\hline
\end{tabular}

Median and minimum-maximum values of viremia and cellular-associated viral loads are indicated for the respective genotypes, HIV-1 RNA in copies/mL, and HIV-1 DNA in copies $/ 10^{6}$ PBMCs. The $p$ values are for median comparisons; N.S.: not significant.

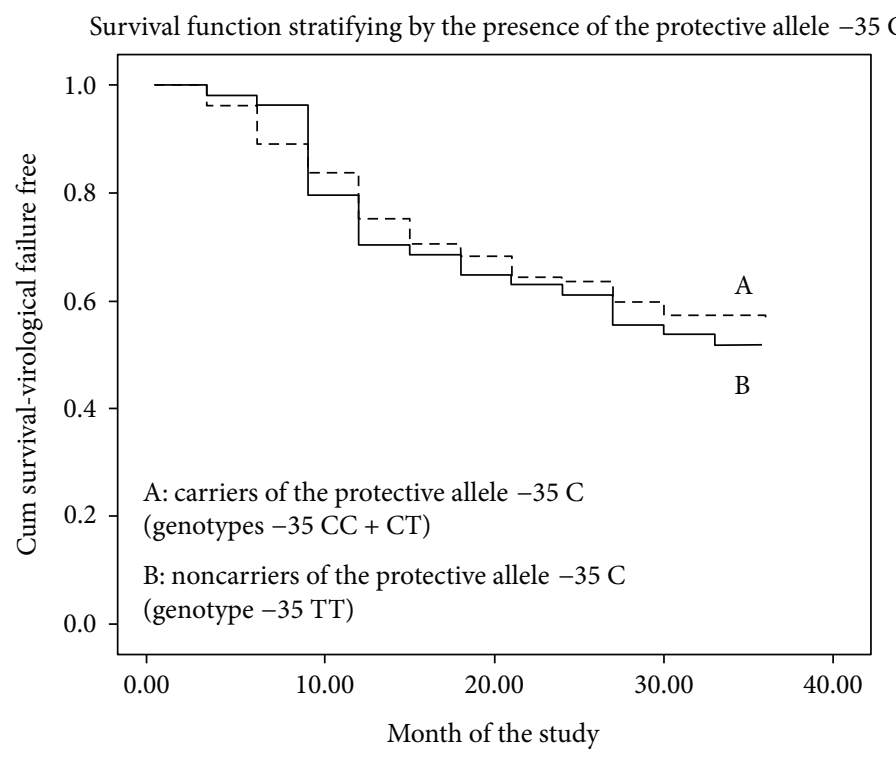

FIgURE 1: Kaplan-Meier estimates of virological failure-free survival during antiviral therapy according to the stratification by the presence of the protective allele -35 C (SNP rs9264942).

TABLE 4: Cox regression model for the virological failure during antiviral therapy.

\begin{tabular}{|c|c|c|c|}
\hline Variable & $\operatorname{HR}(95 \% \mathrm{CI})$ & Endpoint $p(\%)$ & $p$ \\
\hline HIV-1 RNA $\left(\log _{10} \text { copies } / \mathrm{mL}\right)^{\mathrm{a}}$ & $1.86(1.27-2.72)$ & 65.5 & 0.001 \\
\hline HIV-1 DNA $\left(\log _{10} \operatorname{copies} / 10^{6} \text { PBMC) }\right)^{a}$ & $1.74(1.10-2.77)$ & 63.0 & 0.018 \\
\hline Protective allele $-35 \mathrm{C}^{\mathrm{b}}$ & $0.90(0.54-1.50)$ & 47.3 & 0.698 \\
\hline
\end{tabular}

Cox regression model for the virological failure according to the presence of the protective allele - 35 C for the SNP rs9264942 adjusting for HIV-1 RNA levels and HIV-1 DNA levels. Hazard ratio (HR), $95 \%$ confidence intervals (CIs), endpoint $p$ (probability to reach an endpoint, as defined by HR/(1+HR)), and $p$ values are shown. ${ }^{a} \mathrm{HRs}$ calculated for a $1-\log _{10}$ increased. ${ }^{\mathrm{b}} \mathrm{HR}$ calculated for the allele $-35 \mathrm{C}$ as a categorical variable (presence of the allele $-35 \mathrm{C}$ for the $\mathrm{SNP}$ rs9264942[T $>$ C]).

and noncarriers of the allele $-35 \mathrm{C}$ reached the study endpoint (VL $>5000$ copies/mL or CD4 T cells $<250$ cells $/ \mathrm{mL}$ ) without showing differences statistically significant [mean time to the endpoint $=30.0$ months (CI, 27.4-32.5) versus mean time to the endpoint $=29.0$ months $(C I, 25.1-32.9)$, respectively; log-rank test $p=0.616$; Figure 1 ].

A multivariate Cox model analysis was performed by introducing as variable the presence of the protective allele $-35 C$ and covariating with the variables HIV-1 RNA level and HIV-1 DNA level at the baseline pre-ART (Table 4). HIV-1 DNA and HIV-1 RNA were both independently associated with virological failure. The adjusted HR was significant for each $1-\log _{10}$ increase in the baseline HIV1 RNA level (adjusted HR, 1.86 [95\% IC, 1.27-2.72, $p=$ $0.001]$ ) and for each $1-\log _{10}$ increase in the baseline HIV-1 DNA (HR 1.74 [95\% CI, 1.10-2.77, $p=0.018]$ ). The protective allele $-35 C$ was not significantly associated with the virological failure in this model $(p=0.69)$. Adjustment 


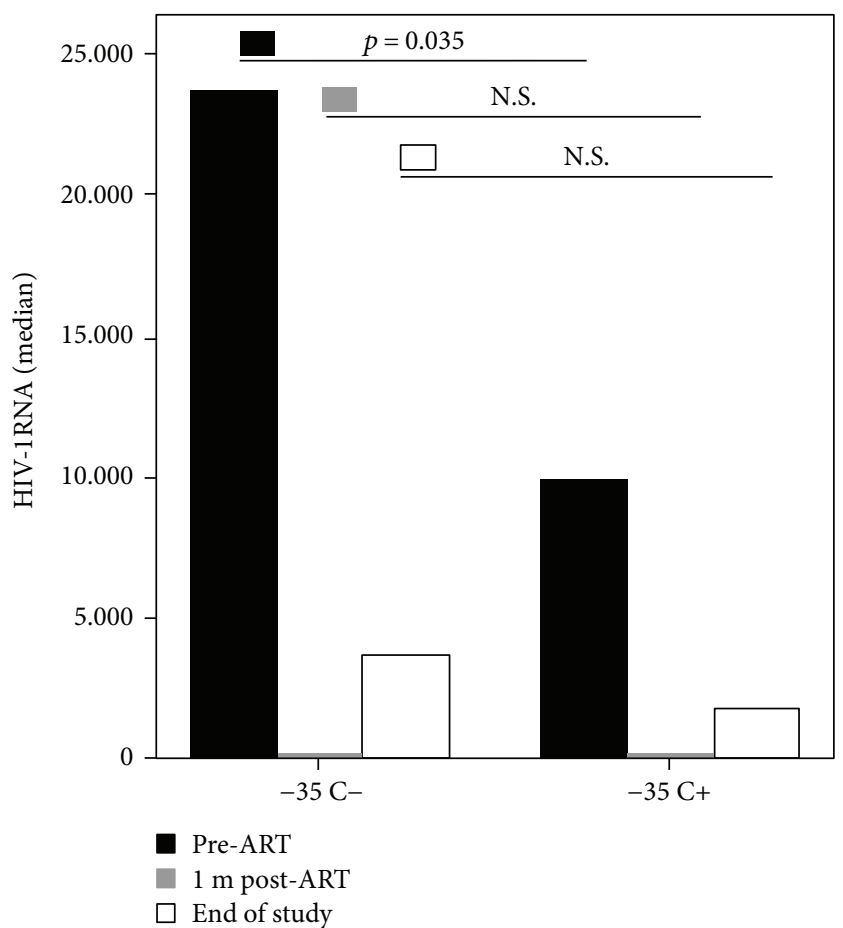

(a)

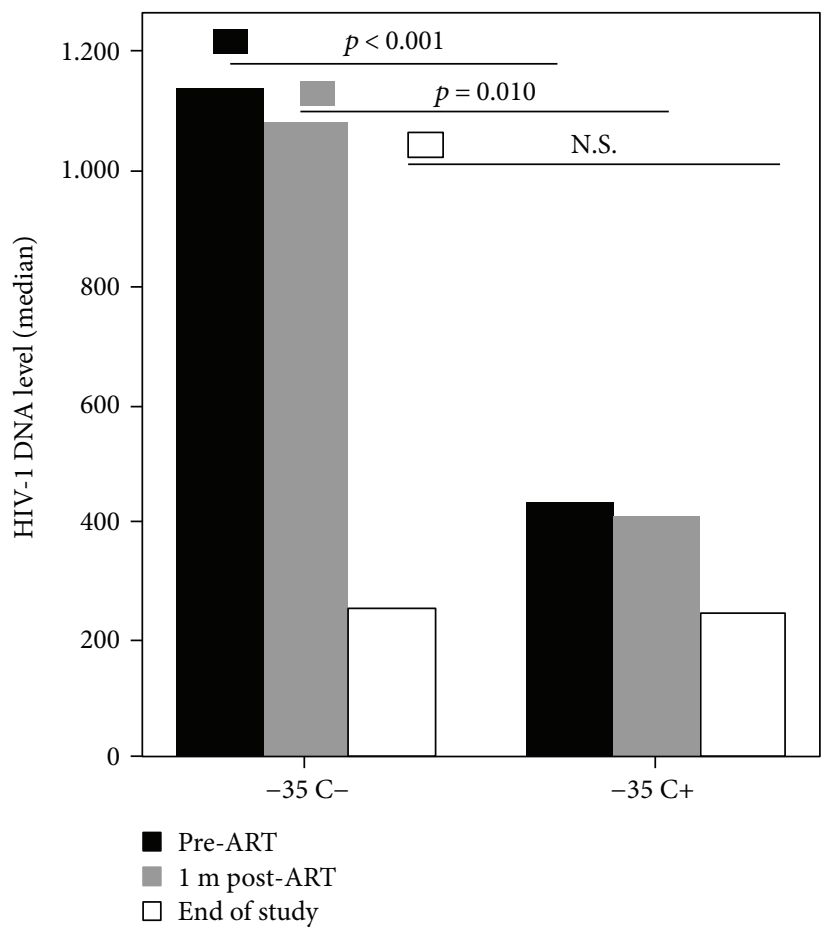

(b)

FIGURE 2: Graph showing the median of HIV-RNA viral load (a) and the median of HIV-1 DNA reservoirs (b) over time (pre-ART, one month post-ART, and at the end of the study) in the patients carrying the protective allele $-35 \mathrm{C}+($ genotypes $\mathrm{CC}+\mathrm{CT})$ and patients noncarrying $-35 \mathrm{C}$ (genotype TT); $p$ values are indicated.

for treatment arms (IMN and IFA), age of patients, and $C C R 5^{+}, H L A-B^{*} 27^{+} / H L A-B^{*} 57^{+}$genotypes affected on the Cox model analysis marginally.

The immunovirological response to the antiretroviral therapy was not significantly different between carriers and noncarriers of the allele $-35 \mathrm{C}$ in each measure of the surrogate markers of disease progression $\left(\mathrm{CD}^{+} \mathrm{T}\right.$ lymphocyte counts and RNA HIV-1 levels measured every three months during 36 months of follow-up). The SNP did not predict $\mathrm{CD} 4{ }^{+} \mathrm{T}$ cell recovery after 36 months on ART.

Finally, although HIV-1 DNA levels, measured retrospectively at the baseline pre-ART and one month postART were significantly different between patients with and without the protective allele, the measurements of HIV-1 DNA levels at the end of the study were not significantly different $(p=0.458)$.

\section{Discussion}

HIV-1 infection exhibits a considerably phenotypic heterogeneity that may be attributed to a complex interplay between viral, environmental, and host genetic factors. Host genes have been extensively explored by several genomewide association studies, which compiled common human genetic variations across diverse continental populations. More than 16 GWAS targeting various HIV-linked phenotypes have been published since 2007 [1-5, 20]. Surprisingly, only the two HIV-1 chemokine coreceptors and HLA loci have exhibited consistent and reproducible statistically significant genetic associations. GWAS focusing on viral load control allowed to identify the association between plasma RNA viral load and the polymorphism rs9264942 located $35 \mathrm{~kb}$ upstream HLA-C. Potential association of this SNP with HIV-1 reservoirs, particularly HIV-1 DNA levels had not been investigated. HIV-RNA level from plasma and HIV-1 DNA level from PBMCs are closely related variables but each one provides slightly different information about the replicative history and spread of the virus [21-23]. Both viral markers do not necessarily show the same trend.

Here, we have retrospectively genotyped the $-35 \mathrm{C} / \mathrm{T}$ polymorphism rs9264942 in $183 \mathrm{HIV}-1$ chronically infected asymptomatic subjects and have confirmed the association of the $-35 C / T$ variant with the HIV-1 viral control. In our untreated patients, the protective allele $-35 C$ is associated with lower viremias as previously demonstrated and also with lower levels of cellular reservoirs of HIV-1 DNA. Even one month posttherapy, when the majority $(78.5 \%)$ of the 183 patients in our cohort showed complete suppression of HIV-1 RNA viremia, they maintained significantly different amounts of HIV DNA load between carriers and noncarriers of the protective allele $-35 \mathrm{C}$. However, the trend for diminished HIV-1 DNA levels did not reach statistically significance at the end of the study STIR-2102, in response to antiviral therapy after thirty-six months on therapy. Carriers and noncarriers of the allele $-35 C$ reached the study endpoint without showing statistically significant differences. Probably the allele $-35 \mathrm{C}$ has a moderate impact on the viral control only in the natural history of the infection, disappearing when treated with antiretrovirals (Figure 2). The mechanisms that regulate HLA-C expression and the link between 
this molecule and HIV infection are still not fully understood. Higher levels of HLA-C associated to the protective allele $-35 \mathrm{C}$ may be important for the viral control in natural history of infection, involving both lower viremias and lower cellular HIV-1 reservoirs, possibly promoting effective CTL recognition and lysis of $\mathrm{HIV}$-infected cells and modulating NK cell activity through the interaction with KIR receptors [24]. Whether HLA-C expression levels are directly responsible for the protective effect of -35 SNP (or 263 insertion/ deletion polymorphism) or the strong linking disequilibrium between the protective variant $-35 C$ and other protective genes in the HLA locus exerts viral control is still unclear. This is extremely difficult to unravel; therefore, we cannot exclude the possibility that some protective HLA-alleles have an effect on the viral control independently or in conjunction with -35 SNP $[20,25]$. Certain HLA variants have been previously associated with viral control such as the protective alleles $H L A-B^{*} 57$ and $H L A-B^{*} 27$ [1-4]. In our study, we have also investigated these alleles (data not shown): eight patients carried the allele $H L A-B^{*} 57$ and other 8 patients carried the allele $H L A-B^{*} 27$. We did not find any protective effect of these alleles on HIV-1 RNA or HIV-1 DNA viral load before or after the antiretroviral treatment. Probably, the statistical power of our study was limited taking into account the low frequency of both $H L A-B^{*} 57$ and $H L A-B^{*} 27$ in our population and the sample size. Therefore, our data cannot demonstrate an additive and independent protective effect of the alleles $H L A-B^{*} 57, H L A-B^{*} 27$, and -35 HLA-C.

Finally, synergistic interactions between loci may affect outcome after infection, as suggested by associations of specific, functionally relevant HLA and KIR variants with HIV disease outcomes and these require further consideration as well. Several association studies have shown that certain HLA/KIR pairs of genotypes (HLA-Bw4/KIR3DL and HLA$B w 4 / K I R 3 D S 1)$ are associated with lower rates of disease progression [26-28]. As HLA-C is the natural ligand for the inhibitory receptors KIR2DL1 and KIR2DL2/3, it would be worthy to study HLA-C group haplotypes $(\mathrm{C} 1 / \mathrm{C} 2)$ in relation with the corresponding polymorphic receptors KIR, in addition to the polymorphism rs9264942. Inhibitory signals derived from the KIR/HLA interactions play a pivotal role in discriminating normal from pathologic tissue and NK cell responses resulting in target infected-cell lysis [29]. To date, it is not clear the relevance of HLA-C levels and haplotypes in combination with different KIR in the HIV viral control.

The design of future studies might consider that the host and viral genetic variation are operating in an interacting system and probably each factor is not in itself sufficient to confer perdurable or complete protection for the disease outcome [30]. Considering the dynamic nature of hostpathogen interactions can shape optimized approaches to long-term HIV-1 management.

\section{Conclusions}

HIV-1 RNA viral load and HIV-1 DNA are both, in our study, variables significantly different between carriers and noncarriers of the protective allele $-35 \mathrm{C}$ before ART. Higher levels of HLA-C associated to the protective allele $-35 \mathrm{C}$ may be important for the viral control in natural history of infection, involving both lower viremias and lower cellular HIV-1 reservoirs, possibly promoting effective CTL recognition and lysis of HIV-infected cells and modulating NK cell activity through the interaction with KIR receptors. This protective effect of the $-35 \mathrm{C}$ allele on viral control was not maintained after the antiretroviral treatment. Probably, this allele has a moderate impact on the viral control only in the natural history of the infection, disappearing when treated with antiretrovirals (Figure 2).

\section{Additional Points}

Highlights. (1) The polymorphism rs9264942 (C/T) impacts on HIV-1 DNA reservoirs. (2) The rs9264942 allele C is associated with lower levels of HIV-1 DNA. (3) This protective effect is observed in untreated patients. (4) The SNP rs 9264942 is not associated with the viral control after 36 months on ART. (5) The SNP rs9264942 do not influence T cell recovery in response to ART

\section{Disclosure}

An earlier version of this work was presented as an abstract at Congreso de la Sociedad Española de Inmunología 2016.

\section{Conflicts of Interest}

The authors declare no conflict of interest.

\section{Authors' Contributions}

Eduardo Fernández-Cruz and Carmen Rodríguez-Sainz conceived and designed the experiments. Laura HerráizNicuesa, Diana Carolina Hernández-Flórez, Lara Valor, and Carmen Rodríguez-Sainz performed the experiments. Eduardo Fernández-Cruz and Carmen Rodríguez-Sainz analyzed the data. Eduardo Fernández-Cruz and Carmen Rodríguez-Sainz wrote the paper.

\section{Acknowledgments}

The authors thank the patients and investigators for contributing the samples studied in the STIR-2102 Study. This work was supported by Fondo de Investigación Sanitaria (FIS), Grants PI 06/1255 and PI 09/2558.

\section{References}

[1] M. P. Martin and M. Carrington, "Immunogenetics of HIV disease," Immunological Reviews, vol. 254, no. 1, pp. 245264, 2013.

[2] S. Limou and J.-F. Zagury, "Immunogenetics: genome-wide association of non-progressive HIV and viral load control: HLA genes and beyond," Frontiers in Immunology, vol. 4, article 118, 2013.

[3] J. Fellay, K. V. Shianna, D. Ge et al., "A whole-genome association study of major determinants for host control of HIV-1," Science, vol. 317, no. 5840, pp. 944-947, 2007. 
[4] The International HIVCS, "The major genetic determinants of HIV-1 control affect HLA class I peptide presentation," Science, vol. 330, no. 6010, pp. 1551-1557, 2010.

[5] J. Fellay, D. Ge, K. V. Shianna et al., "Common genetic variation and the control of HIV-1 in humans," PLoS Genetics, vol. 5, no. 12, article e1000791, 2009.

[6] R. Apps, Y. Qi, J. M. Carlson et al., "Influence of HLA-C expression level on HIV control," Science, vol. 340, no. 6128, pp. 87-91, 2013.

[7] S. Kulkarni, R. Savan, Y. Qi et al., "Differential micro RNA regulation of HLA-C expresión and its association with HIV control," Nature, vol. 472, no. 7344, pp. 495-498, 2011.

[8] T. W. Corrah, N. Goonetilleke, J. Kopycinski et al., "A reappraisal of the relationship between the HIV-1-protective single nucleotide polymorphism $35 \mathrm{~kb}$ upstream of the HLA-C gene and surface HLA-C expression," Journal of Virology, vol. 85, no. 7, pp. 3367-3374, 2011

[9] R. Thomas, R. Apps, Y. Qi et al., "HLA-C cell surface expression and control of HIV/AIDS correlate with a variant upstream of HLA-C," Nature Genetics, vol. 41, no. 12, pp. 1290-1294, 2009.

[10] E. J. Adams and P. Parham, "Species-specific evolution of MHC class I genes in the higher primates," Immunological Reviews, vol. 183, no. 1, pp. 41-64, 2001.

[11] A. M. Older Aguilar, L. A. Guethlein, E. J. Adams, L. Abi-Rached, A. K. Moesta, and P. Parham, "Coevolution of killer cell Ig-like receptors with HLA-C to become the major variable regulators of human NK cells," Journal of Immunology, vol. 185, no. 7, pp. 4238-4251, 2010.

[12] D. Snary, C. J. Barnstable, W. F. Bodmer, and M. J. Crumpton, "Molecular structure of human histocompatibility antigens: the HLA-C series," European Journal of Immunology, vol. 7, no. 8, pp. $580-585,1977$.

[13] J. A. McCutcheon, J. Gumperz, K. D. Smith, C. T. Lutz, and P. Parham, "Low HLA-C expression at cell surfaces correlates with increased turnover of heavy chain mRNA," The Journal of Experimental Medicine, vol. 181, no. 6, pp. 20852095, 1995.

[14] A. Neisig, C. J. M. Melief, and J. Neefjes, "Reduced cell surface expression of HLA-C molecules correlates with restricted peptide binding and stable TAP interaction," Journal of Immunology, vol. 160, pp. 171-179, 1998.

[15] S. Le Gall, L. Erdtmann, S. Benichou et al., "Nef interacts with the 1 subunit of clathrin adaptor complexes and reveals a cryptic sorting signal in MHC I molecules," Immunity, vol. 8, no. 4, pp. 483-495, 1998.

[16] E. Fernandez-Cruz, S. Moreno, J. Navarro et al., "Therapeutic immunization with an inactivated HIV-1 Immunogen plus antiretrovirals versus antiretrovirals therapy alone in asymptomatic HIV-infected subjects," Vaccine, vol. 22, no. 23.24, pp. 2966-2973, 2004.

[17] L. Valor, J. Navarro, J. Carbone et al., "Immunization with an HIV-1 immunogen induces CD4+ and CD8+ HIV-1-specific polyfunctional responses in patients with chronic HIV-1 infection receiving antiretroviral therapy," Vaccine, vol. 26, no. 22, pp. $2738-2745,2008$

[18] C. Rodríguez-Sáinz, R. Ramos, L. Valor et al., "Prognostic value of peripheral blood mononuclear cell-associated HIV-1 DNA for virological outcome in asymptomatic HIV-1 chronic infection," Journal of Clinical Virology, vol. 48, no. 3, pp. 168$172,2010$.
[19] P. Grambsch and T. Therneau, "Proportional hazards tests and diagnostics based on weighted residual," Biometrika, vol. 81, no. 3, pp. 515-526, 1994.

[20] P. J. McLaren, C. Coulonges, I. Bartha et al., "Polymorphisms of large effect explain the majority of the host genetic contribution to variation of HIV-1virus load," PNAS, vol. 112, no. 47, pp. 14658-14663, 2015.

[21] V. Avettand-Fèncël, L. Hocqueloux, J. Ghosn et al., “Total HIV-1 DNA, a marker of viral reservoir dynamics with clinical implications," Clinical Microbiology Reviews, vol. 29, no. 4, pp. 859-880, 2016.

[22] R. Banga, F. A. Procopio, and M. Perreau, "Current approaches to assess HIV-1 persistance," Current Opinion in HIV and AIDS, vol. 11, no. 4, pp. 424-431, 2016.

[23] M. J. Churchill, S. G. Deeks, D. M. Margolis, R. F. Siliciano, and R. H. I. V. Swanstrom, "Reservoirs: what, where and how to target them," Nature Reviews. Microbiology, vol. 14, no. 1, pp. 55-60, 2016.

[24] M. Sips, Q. Liu, M. Draghi et al., "HLA-C levels impact natural killer cell subset distribution and function," Human Immunology, vol. 77, no. 12, pp. 1147-1153, 2016.

[25] M. Carrington, A. A. Bashirova, and P. J. McLaren, "On stand by: host genetics of HIV control," AIDS, vol. 27, no. 18, pp. 2831-2839, 2013.

[26] M. P. Martin, X. Gao, J.-H. Lee et al., "Epistatic interaction between KIR3DS1 and HLA-B delays the progression to AIDS," Nature Genetics, vol. 31, no. 4, pp. 429-434, 2002.

[27] K. Pelak, A. C. Need, J. Fellay et al., "Copy number variation of KIR genes influences HIV-1 control," PLoS Biology, vol. 9, no. 11, article e1001208, 2011.

[28] M. P. Martin, Y. Qi, X. Gao et al., "Innate partnership of HLA$\mathrm{B}$ and KIR3DL1 subtypes against HIV-1," Nature Genetics, vol. 39, no. 6, pp. 733-740, 2007.

[29] J. Hens, W. Jennes, and L. Kestens, "The role of NK cells in HIV-1 protection: autologous, allogeneic or both?," AIDS Research and Therapy, vol. 13, no. 1, article 15, 2016.

[30] P. J. McLaren and J. Fellay, "Human genetic variation in HIV disease: beyond genome-wide association studies," Current Opinion in HIV and AIDS, vol. 10, no. 2, pp. 110-115, 2015. 


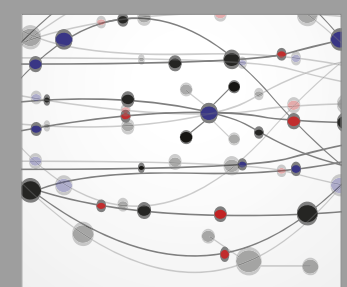

The Scientific World Journal
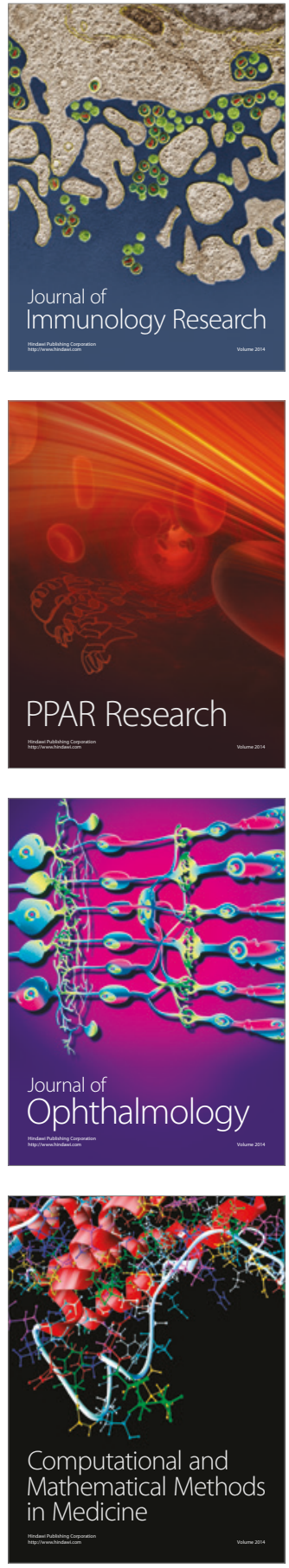

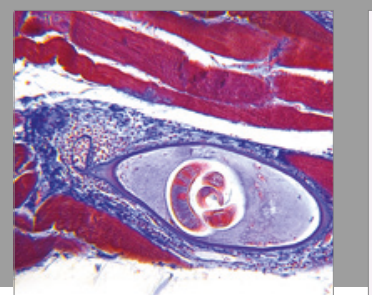

Gastroenterology Research and Practice
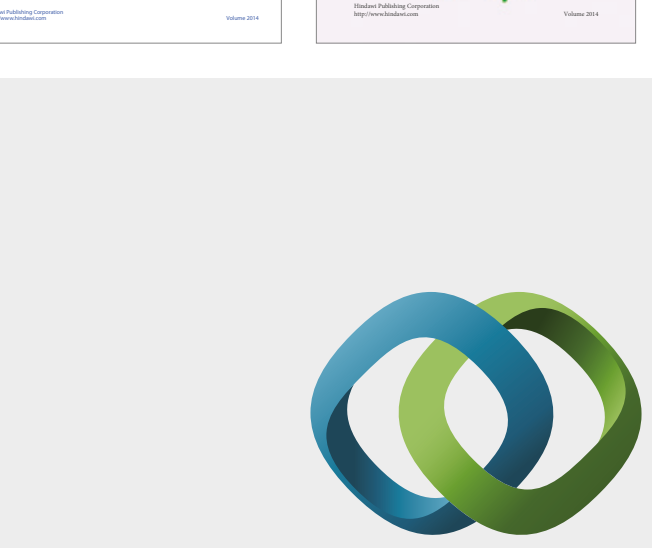

\section{Hindawi}

Submit your manuscripts at

https://www.hindawi.com
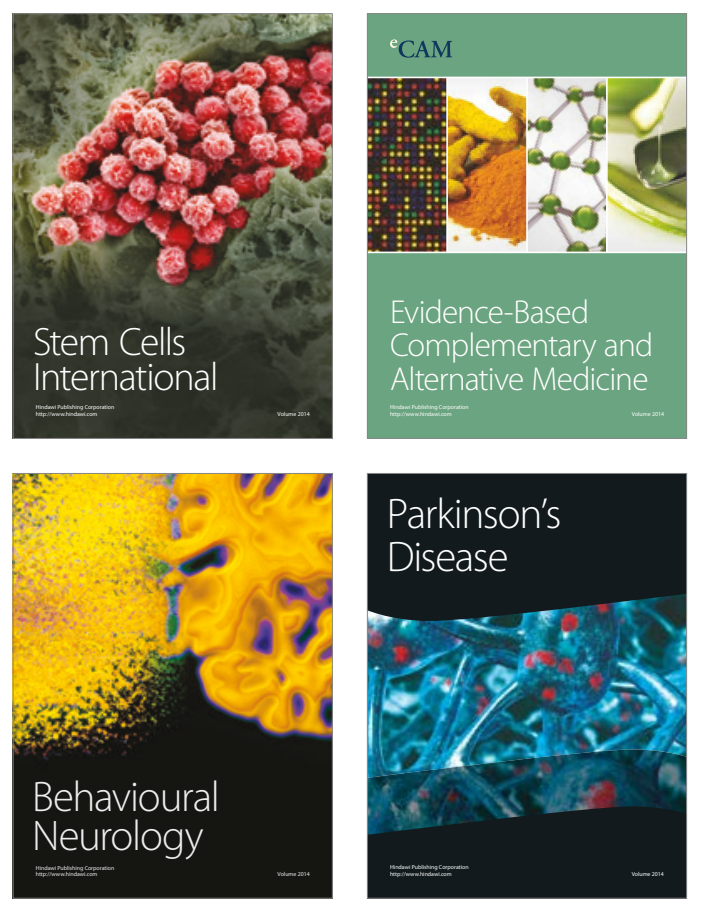
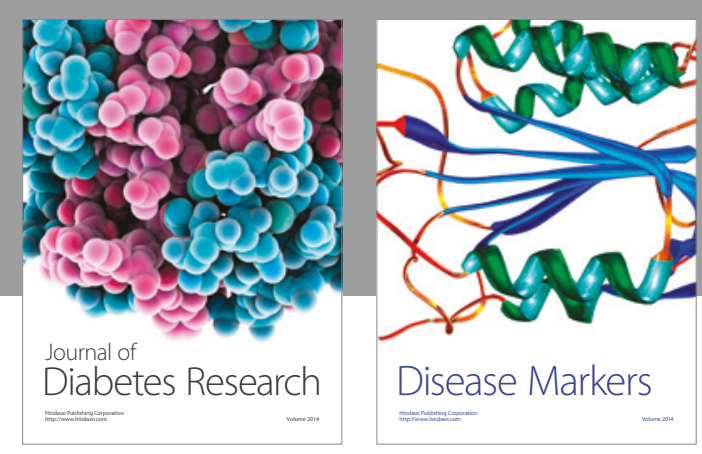

Disease Markers
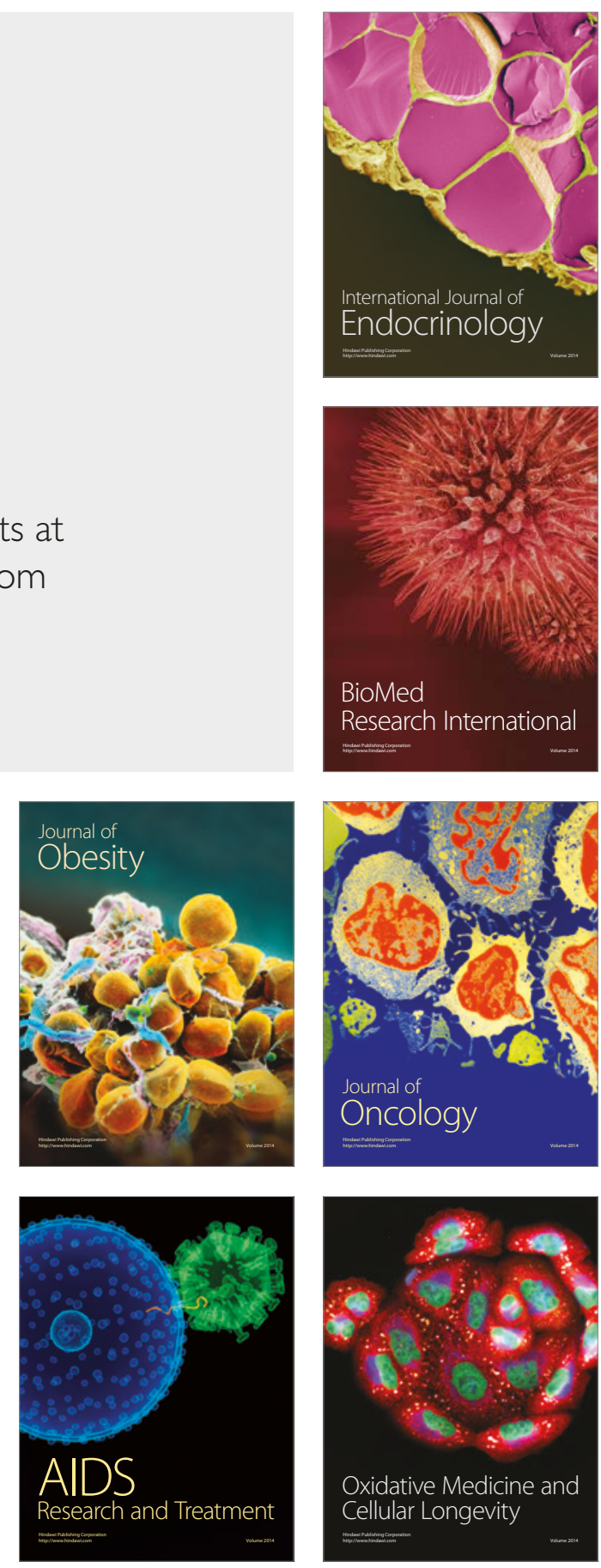